\title{
Towards the computer visualization of electrodynamics education for undergraduates major in optics
}

Jing Liu, Minhao Gao, Yansong Fan

Jing Liu, Minhao Gao, Yansong Fan, "Towards the computer visualization of electrodynamics education for undergraduates major in optics," Proc. SPIE 10452, 14th Conference on Education and Training in Optics and Photonics: ETOP 2017, 104520A (16 August 2017); doi: 10.1117/12.2264607

SDIE Event: 14th Conference on Education and Training in Optics and Photonics, ETOP 2017, 2017, Hangzhou, China 


\title{
Towards the computer visualization of electrodynamics education for undergraduates major in optics
}

\author{
Liu Jing, Gao Minhao, Fan Yansong \\ College of Optoelectronic Science and Engineering, National University of Defense Technology, \\ Changsha 410073, China
}

\begin{abstract}
We present a general review on using computer visualization technology to assist teaching electrodynamics for undergraduates in several Chinese universities. Based on our own teaching activities during the past decade in National University of Defense and Technology, China, we propose and discuss the necessity of computer visualization in electrodynamics course teaching for undergraduates major in optics for the first time. Then we will show how to help students to comprehend fundamental concepts and to understand the effect of parameters on core physical quantities through some teaching designs. At last, specified content inside the course that are suitable to be assisted by computer visualization are demonstrated.
\end{abstract}

Keywords: Electrodynamics education, Computer visualization, Undergraduates major in optics

\section{INTRODUCTION}

According to the "Outline of China's National Plan for Medium and Long-term Education Reform and Development (2010-2020)", raising the education quality is appointed at the heart of task for the present Chinese higher education reform and development ${ }^{[1]}$. Consequently, course teaching, as the most important activity in the university, has become top-concerned and widely-discussed subject. In National University of Defense Technology, China, theoretical physics courses including theoretical mechanics, thermodynamics and statistical physics, electrodynamics, and quantum mechanics are setup for undergraduates major in optics. Practice tell us that those action helps to reinforce the physical fundamentals and students benefit from that when learning laser principles and optoelectronics in the next stage. However, in recent years, course teaching is serious challenged by the fact that class hours for theory courses are significantly decreased, while simultaneously students are required to master more professional basics and practical ability, which weakens the effect of original design. In this case, novel teaching strategies should be proposed and implemented.

The "Decades of development planning of educational informationization (2011-2020)" released by Chinese Ministry of Education (MOE) has classified both the promotion of integrating information technology with education, and the cultivating students' learning ability under the informationization environment as important development tasks ${ }^{[2]}$. Employing computer visualization technology to assist teaching has been suggested by educational experts and scholars for a long time, and using computer visualization technology to assist teaching electrodynamics is a promising route.

This paper briefly review these practices performed in several Chinese universities. Based on our own teaching activities during the past decade in National University of Defense and Technology, China, we firstly propose and discuss the

14th Conference on Education and Training in Optics and Photonics: ETOP 2017, edited by Xu Liu,

Xi-Cheng Zhang, Proc. of SPIE Vol. 10452, 104520A · C 2017 ICO, IEEE, OSA, SPIE

CCC code: $0277-786 \mathrm{X} / 17 / \$ 18 \cdot$ doi: $10.1117 / 12.2264607$

Proc. of SPIE Vol. 10452 104520A-1 
necessity of computer visualization in electrodynamics course teaching for undergraduates major in optics. Some teaching designs examples, such as the eigen modes of guiding electromagnetic waves, Cerenkov radiation, and Fresnel formulae, are given to show how to help students to comprehend fundamental concepts and to understand the effect of parameters on key physical quantities. In addition, students could also benefit from this route and implement the mastered method for their further study in other course learning such as Laser Principles and Optoelectronics as well as scientific research. At last, specified content inside the course that are suitable to be assisted by computer visualization are demonstrated.

\section{NECESSITY OF COMPUTER VISUALIZATION IN ELECTRODYNAMICS}

With the rapid development of computer technology, educators in domestic universities have advocated and began to apply computer visualization technology to improve teaching quality as early as 2000 years or $\mathrm{so}^{[3-8]}$. Some commercial software, such as MATLAB and ANYSIS have been widely used in the courses of General physics, especially in Electromagnetics. With the help of powerful visualization technology in these software, the intuitive and vivid presentation form made students acquire a better understanding of the abstract concepts. Besides, it trained their consciousness and ability of solve actual physical problems with the aid of computers.

However, their similar applications in Classical Electrodynamics have been rarely discussed. In fact, the transmission and radiation of electromagnetic wave in the course are more complicated, which are more in need of computer visualization technology. During the teaching practice, we found that extensive and intensive discussions can be well initiated during the students by use of computer visualization technology in class presentation, which is significant and effective for inspiring their deep thinking.

Classical Electrodynamics is a compulsory subject for undergraduates major in optics in our university. It is also their first course raising from general subjects such as math and physics to Optics specialized courses in the third academic year. Polarization, guide, transmission and radiation of electromagnetic wave taught in this course are reserved for our students as a priori knowledge for their following subjects, like physical optics, Principle of Laser and Photo electricity Technology.

\section{TYPICAL TEACHING DESIGNS}

\subsection{Magnetic Fields of Solenoid, Permanent Magnet and Superconductor}

The teaching of electromagnetic theory is generally started from the electrostatic field, and students are often unfamiliar with the abstract but important concept of "field". The key is to get students to the essence of field, which is distributed continuously in a certain space range. Our first teaching step is to visualize the electric and magnetic lines to demonstrate the space distribution of field.

The static magnetic field in an electric vacuum device generated by 5 group of coils are shown in Fig1. They are used in an electric vacuum device, which can radiate electromagnetic wave with central frequency around $28 \mathrm{GHz}$, guiding an intense relativistic electron beam. 

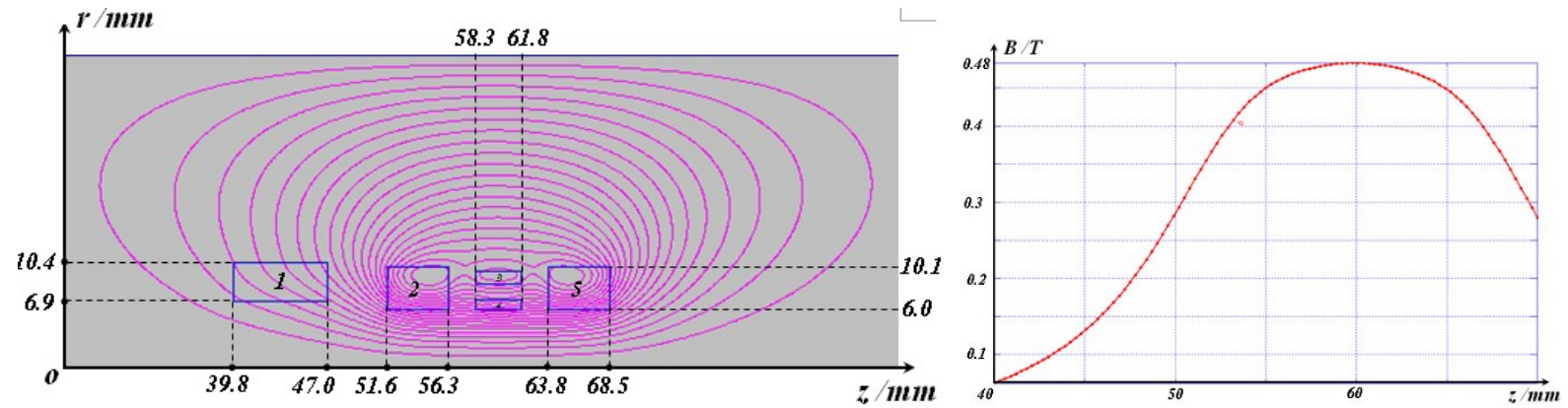

Figure 1 Magnetic field of 5 groups of solenoid

Figure 2 gives the period static magnetic field driven by a group of permanent magnets, which are used in another kind of electric vacuum device.
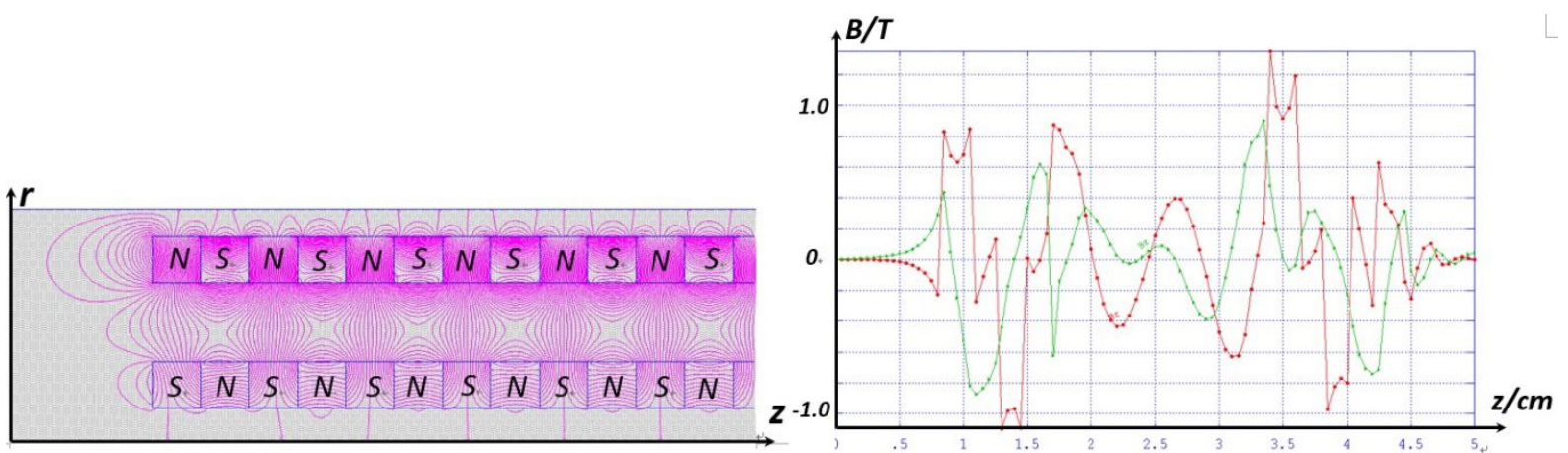

Figure 2 Partial magnetic field of periodic permanent magnets

\subsection{Propagation of Electromagnetic Waves through Homogeneous Media}

Fresnel's Formula presents the reflection and refraction of electromagnetic waves at a plane interface between dielectrics. It is introduced to students usually in Optics at first and also can be derived from Maxwell equations in detail in Electrodynamics.

These distinct phenomena, like total reflection, Brewster Law and polarization, as well as the influences among relevant parameters can be demonstrated intuitively and clearly.
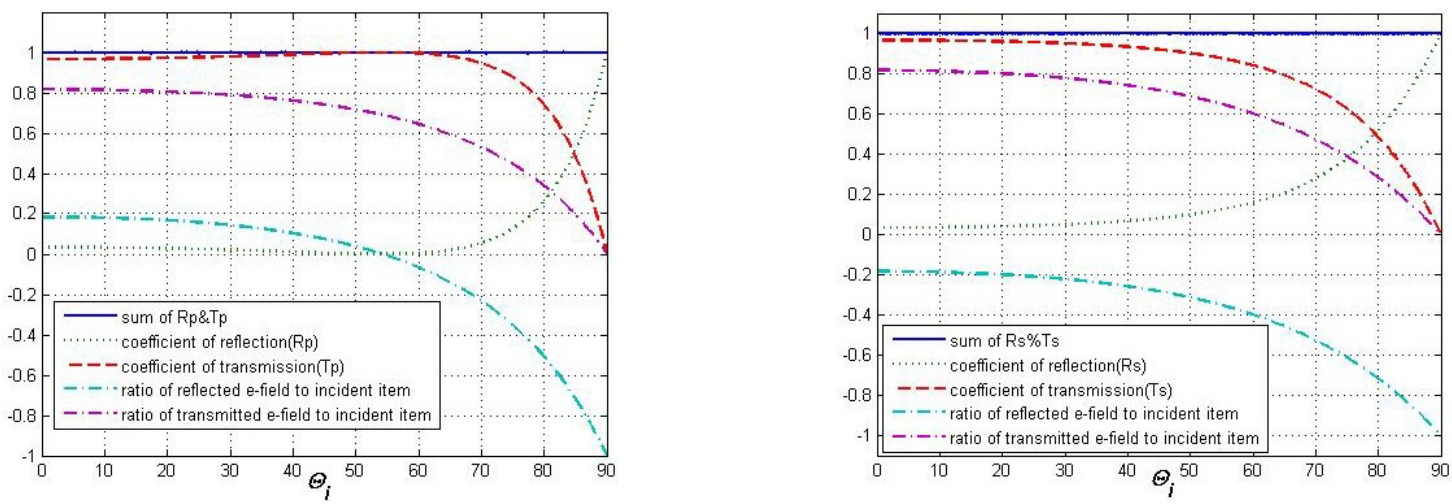

Figure 3 Reflection and refraction of electromagnetic waves at a plane interface from denser to thinner media (left)S- Polarized Light and (right) P-Polarized Light 
Figure 3 shows that (1) When the incident angle closes $54^{\circ}$, the reflection coefficient of P-Polarized Light reduces to zero but that of S-Polarized Light is nonzero. So the electric field of the reflected light is perpendicular to the plane of incidence, namely the reflected light is linearly polarized light. This is the famous Brewster Law of Optics and this correspondent incident angle is called Brewster Angle. (2) When the incident angle increases to more than54 ${ }^{\circ}$, the electric fields of incident and reflected light become to in opposite phase. That is to say there is a half wave loss. (3) For S-Polarized Light, there is not Brewster Angle, and the electric fields of incident and reflected light are in opposite phase all the time, namely the half wave loss always exists. (4) Sum of the reflection coefficient and transmission coefficient for both P-Polarized Light and S-Polarized Light identically equal to one.
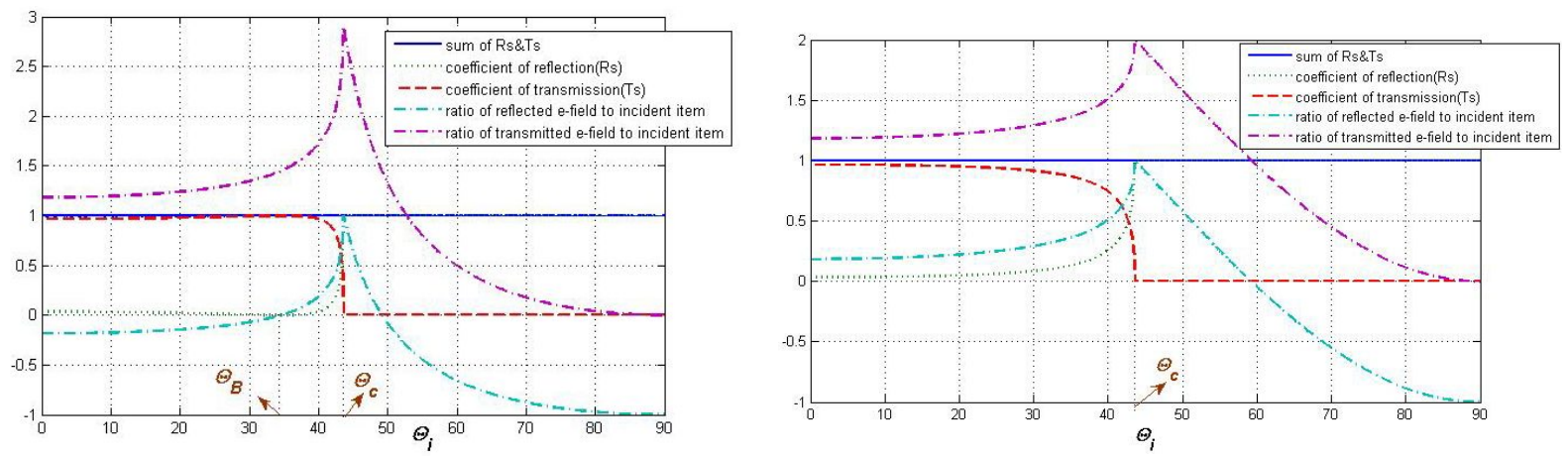

Figure 4 Reflection and refraction of electromagnetic waves at a plane interface from thinner to denser media

(left)S- Polarized Light and (right) P-Polarized Light

Figure 4 shows that (1) there still exists Brewster Angle for S- Polarized Light only. (2) Sum of the reflection coefficient and transmission coefficient for both P-Polarized Light and S-Polarized Light identically equal to one. (3) for both SPolarized and P-Polarized light there is a critical angle, which the incident angle closes to, the reflected light disappears. This is the total reflection we all know well.

\subsection{Modes in Waveguides and Optical Fibers}

When electromagnetic waves are propagated in guided systems, the normal mode expiation for an arbitrary field in a waveguide or optical fibers is widely applied. On the other hand, this is also very difficult for most students because the modes are abstract and complicated. The electric field distribution of the basic low-order modes can be given in more vivid way.

Figure 5 shows the distribution of electric fields for $\mathrm{TE}_{10}$ mode in rectangular waveguide and Figure 6 gives that of $\mathrm{TE}_{11}$ and $\mathrm{TM}_{01}$ modes in cylindrical waveguide. 

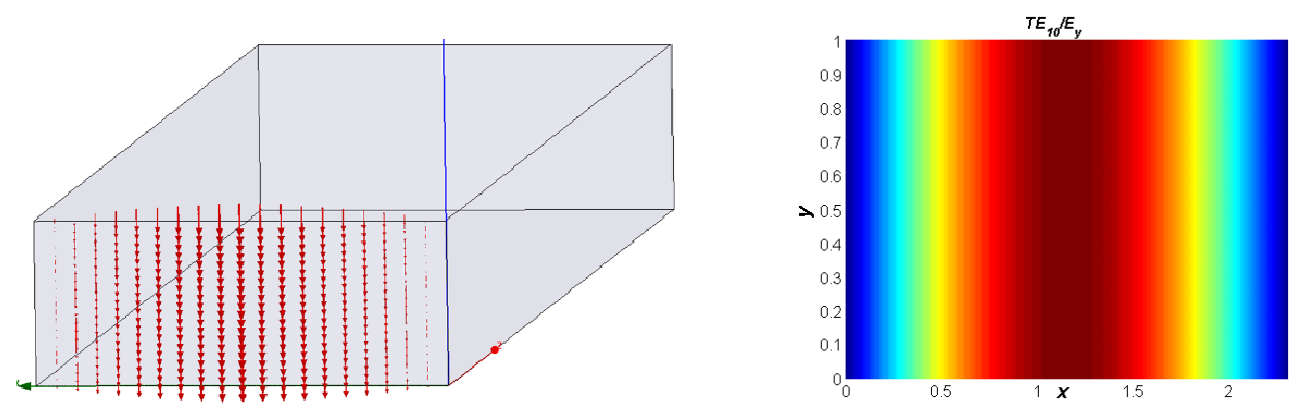

Figure 5 The distribution of electric fields for $\mathrm{TE}_{10}$ mode in rectangular waveguide
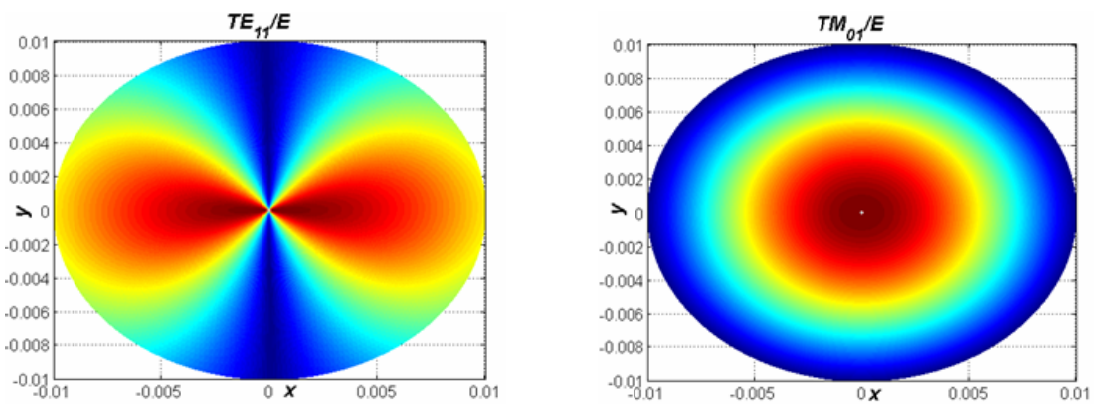

Figure 6 The distribution of electric field for $\mathrm{TE}_{11}$ and $\mathrm{TM}_{01}$ modes in cylindrical waveguide

Figure 7 presents the $2 \mathrm{D}$ and $3 \mathrm{D}$ distributions of light intensity for $\mathrm{LP}_{01}$ mode in optical fiber and Figure 8 displays that for $\mathrm{LP}_{11}$ mode in optical fiber.
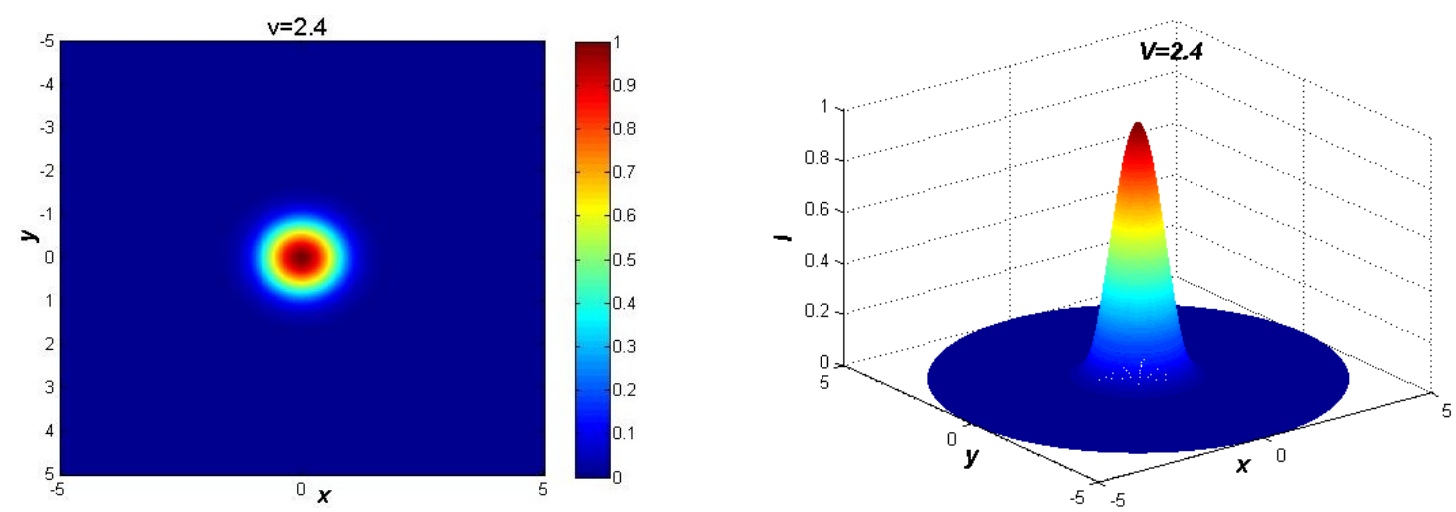

Figure 7 The $2 \mathrm{D}$ and $3 \mathrm{D}$ distributions of light intensity for $\mathrm{LP}_{01}$ mode in optical fiber 

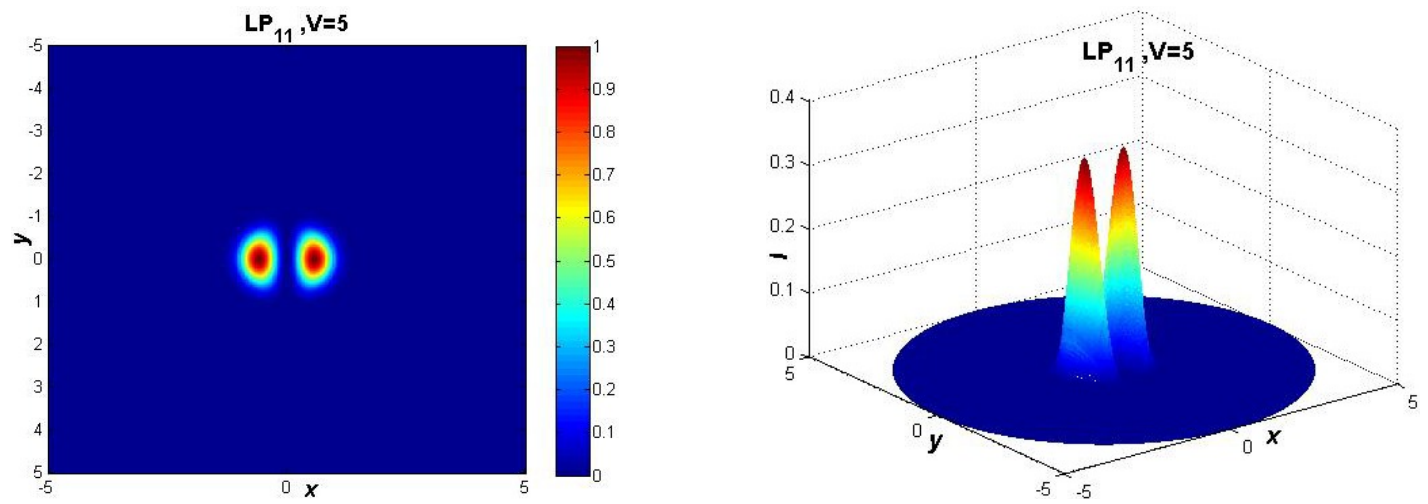

Figure 8 The 2D and 3D distributions of light intensity for $\mathrm{LP}_{11}$ mode in optical fiber

\subsection{Radiations}

In the discussion on the emission of radiation by localized system of oscillating charge and current densities, students often fall into confusion with the complicated and abstract models. The space distribution of radiation intensity of some fundamental radiant systems can be given to help students form clear physical images.

The space distributions of radiation intensity of electric dipole, magnetic dipole and electric quadruple are given in Figure 9 to Figure 11.
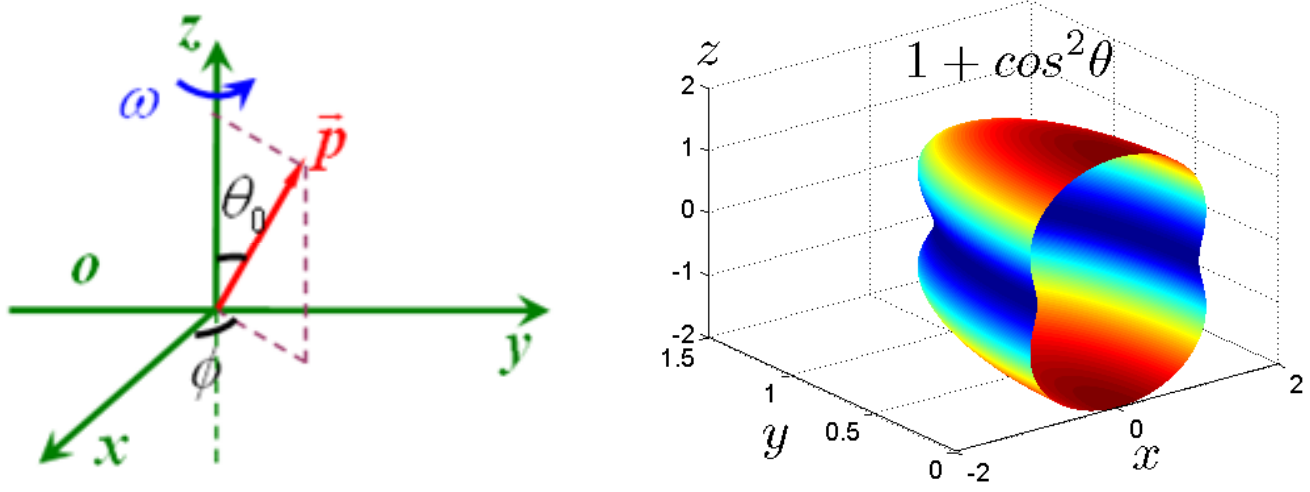

Figure 9 Electric dipole rotating along $\mathrm{z}$ axis with fixed included angle and speed and its space distribution of radiation intensity 

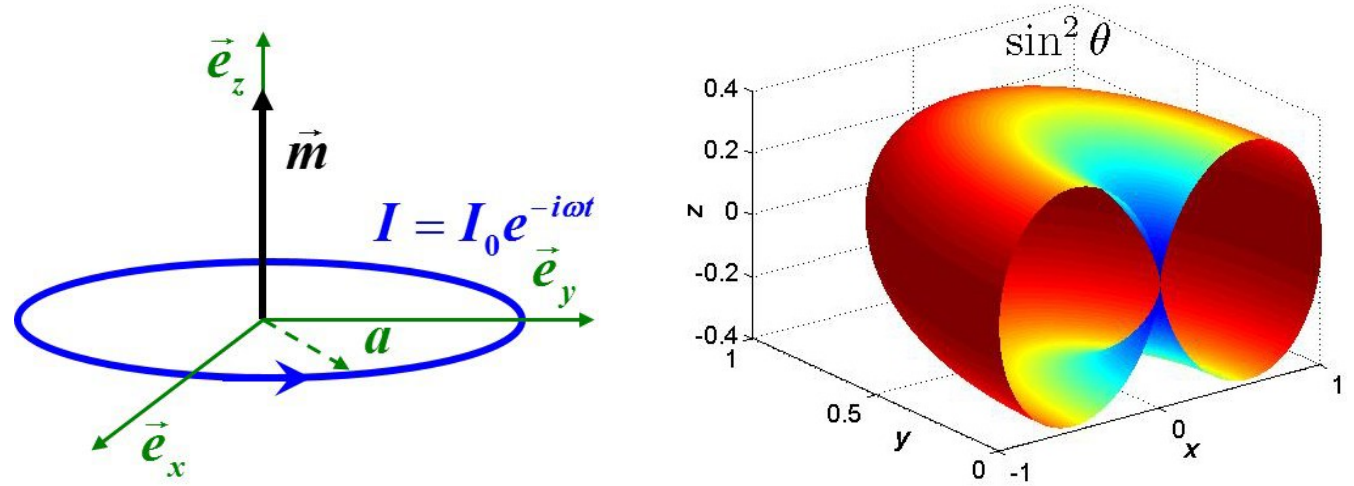

Figure 10 Circle antenna and its magnetic dipole radiation distribution
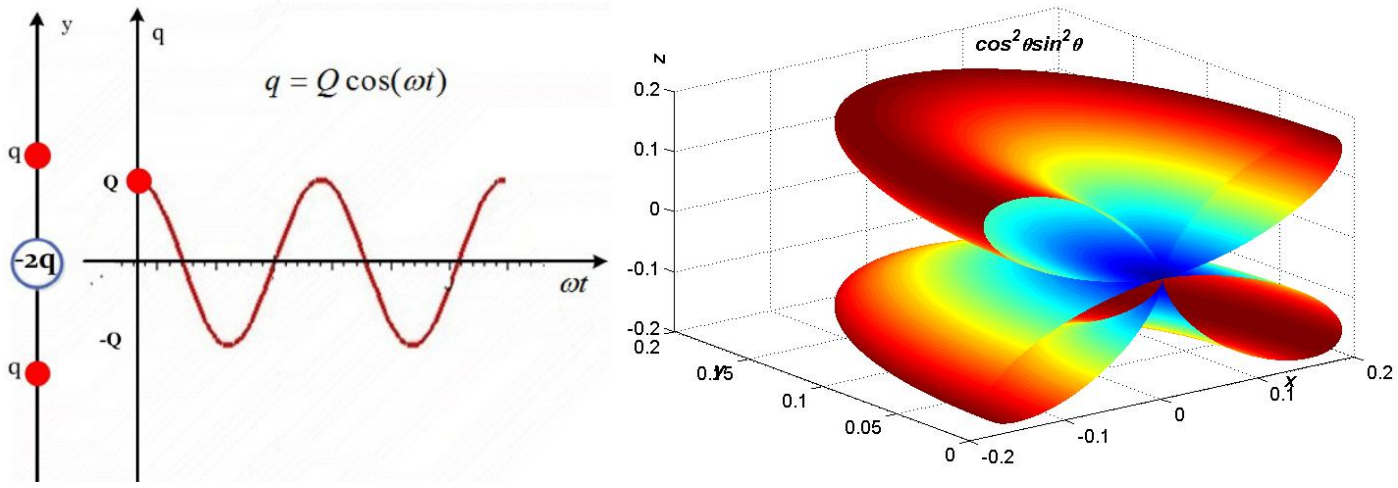

Figure 11 Time-varying positive charges and its electric quadrupole radiation pattern

Besides, the center-fed thin linear antenna is the simplest mode, which approaches the practical engineering application. Figure 12 and Figure 13 give radiation patterns of short antenna, half-wavelength antenna and full-wavelength antenna respectively, as well as their comparison.
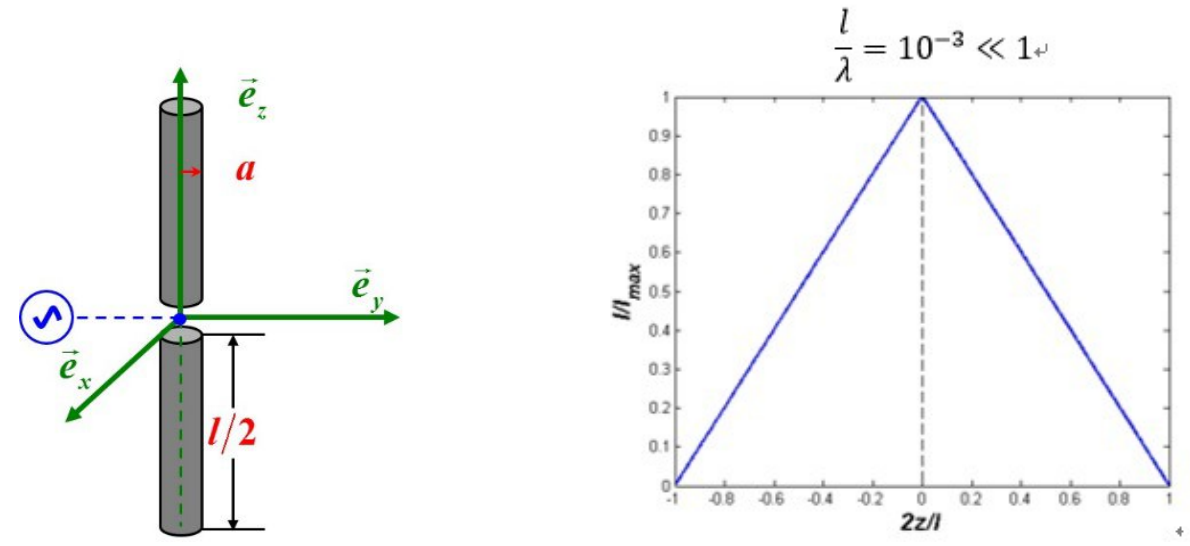

Figure 12 Sketch of short center-fed thin linear antenna and its current distribution 

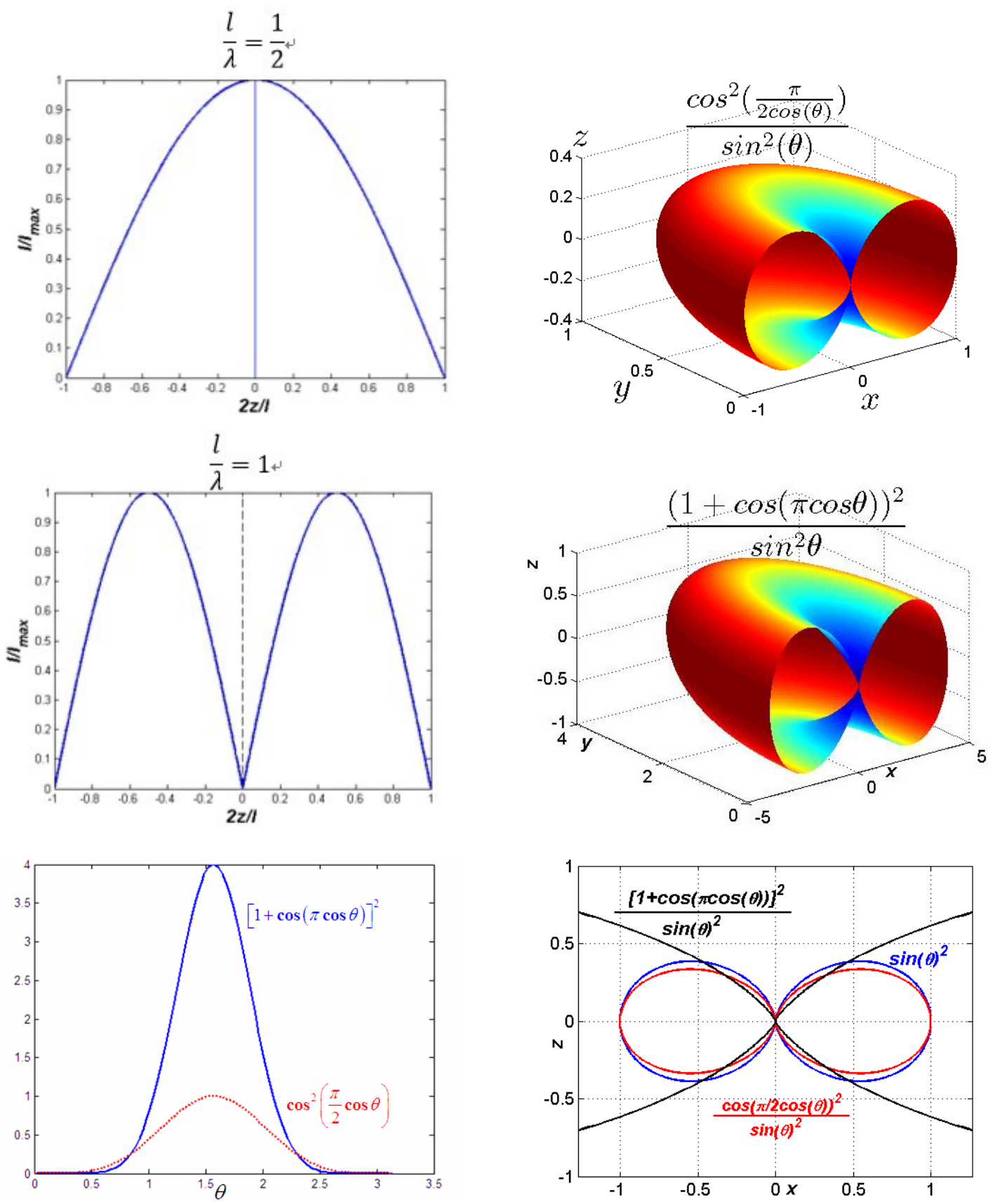

Figure 13 Half-wavelength antenna and full-wavelength antenna and their radiation patterns

Besides, in real engineering experiments, the features of more completed radiate systems are given by aid of some commercial software, such as CST(Computer Simulation Technology), HFSS(High Frequency Structure Simulator) and COMSOL Multiphysics, etc. In the course teaching, we try to introduce some classical simulated results, as shown in Figure14. 

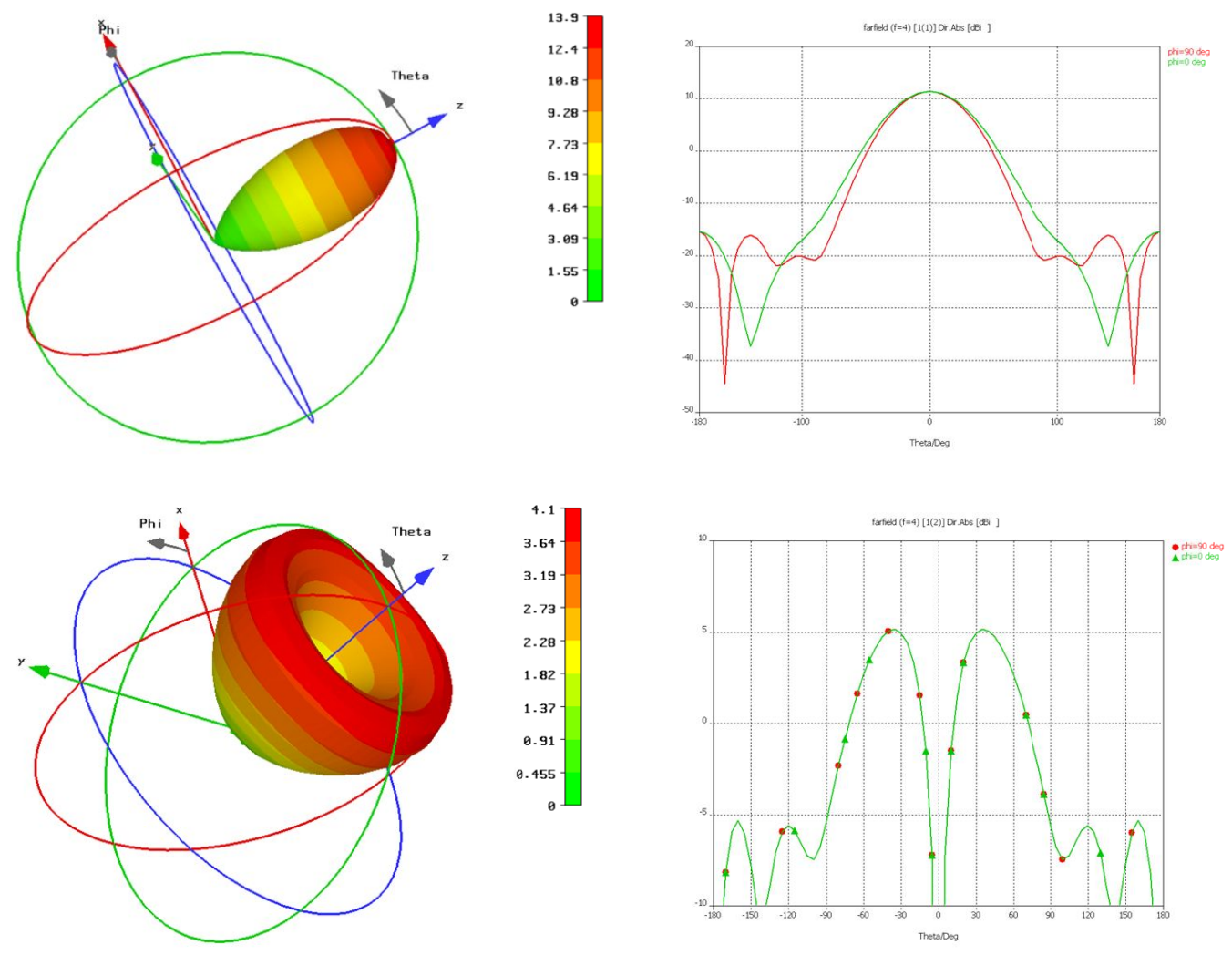

Figure 14 The 3D and 2D directional diagram for radiated (up) $\mathrm{TE}_{11}$ and (down) $\mathrm{TM}_{01}$ modes in space

Figure 14 shows that energy concentrates on the center symmetrical axis for $\mathrm{TE}_{11}$ mode but for $\mathrm{TM}_{01}$ mode it disperses uniformly as a ring around the center symmetrical axis. Therefore, the former is preferred in practical application.

\section{CONCLUSION}

In this article, the application of computer visualization in our Electrodynamics teaching practice are discussed in detail. Spatial distribution of vector field line, the variation of physical quantities with some parameters, the spatial distribution of electric field strength and radiation pattern are demonstrated through specific examples. Combined with the scientific research content of the educators themselves, the means of computer visualization will become more and more abundant, and the demonstration of the material will be more intuitionistic and visual. This also conforms to the trend of make use of images and videos instead of text in the development of internet.

\section{REFERENCES}

[1] http://www.moe.edu.cn/publicfiles/business/htmlfiles/moe/moe_2862/201010/109029.html

[2] http://www.moe.edu.cn/publicfiles/business/htmlfiles/moe/s3342/201203/133322.html

[3] Li Jianfang, Application of scientific visualization technology in Physics Teaching [J], College physics, 1998, 17(9): 38-39. (in chinese) 
[4] Zhao Xiaolong, Wang Yuping and Bao Lihong, Visual simulation design on electrostatic problem in electromagnetism [J], Physics and Engineering, 2012, 22(1):28-31. (in chinese)

[5] Gao Cuiyun and Wang Lili, Applying MATLAB in teaching of electromagnetism for calculation and visualization [J], Journal of Electrical \& Electronic Engineering Education, 2006, (2):90-92. (in chinese)

[6] Wang Shunzhi, Xu Hongxia and Liu Ye, Visual analogy teaching of gravitational field and electrostatic field [J], Physics and Engineering, 2014, 24(s2):48-50. (in chinese)

[7] Liu Qunying, Application of MATALB language in electric-magnetism of college physics [J], Journal of Western Chongqing University (Nature Science Edition), 2005,4(2):19-22. (in chinese)

[8] Ou Pan, Advanced optical simulation (MATLAB Edition)[M], Beijing University of Aeronautics and Astronautics Press, 2011. 